\title{
Revista \\ Brasileira

\section{Análise da atuação do enfermeiro na gerência de unidades básicas de saúde}

\author{
Analysis of managerial work of the nurse in basic health units \\ Análisis del trabajo gerencial del enfermero en unidades básicas de salud
}

\section{Marcelo Costa Fernandes', Adriana Sousa Barros', Lucilane Maria Sales da Silva', Maria de Fátima Bastos Nóbrega", Maria Rocineide Ferreira da Silva', Raimundo Augusto Martins Torres ${ }^{1}$}

'Universidade Estadual do Ceará. Departamento de Enfermagem. Fortaleza, CE

"Universidade Estadual do Ceará. Hospital Universitário Walter Cantídeo. Fortaleza, CE

Submissão: 03/01/2009

Aprovação: 05/01/2010

\section{RESUMO}

Objetivou-se analisar o trabalho do enfermeiro gerente, conhecer suas ações, verificar a importância atribuída ao planejamento e identificar fatores que interferem na gerência. Pesquisa descritiva, realizada em 10 unidades básicas de saúde de Fortaleza, CE com 10 enfermeiros, selecionados por ocuparem cargos de gerentes. A coleta dos dados deu-se em janeiro de 2008, através de entrevista estruturada. Desses a maioria era do sexo feminino na faixa etária de 37 a 57 anos. Para sete dos enfermeiros gerentes a gestão de pessoas e o planejamento é o mais importante. Relataram dificuldades Quanto à composição incompleta das eQuipes, falta de capacitação profissional e carência de recursos financeiros. Essa pesQuisa reúne informações relevantes para conhecimento e análise das ações gerenciais do enfermeiro na atenção básica.

Descritores: Enfermagem; Gerência; Unidade básica de saúde.

\section{ABSTRACT}

The purpose was to analyse the managerial work of the nurse, to know nurses' actions, to verify the importance towards the planning and to identify some factors that interfere in the management. A descriptive research was accomplished in 10 basic health units in Fortaleza, CE, Brazil, with 10 nurses. Data collection was carried aout in january 2008, by means of a structured interview. Most of the nurses were female between 37 and 57 years-old. For seven nurses the management of people and the planning are the most important aspects. Some difficulties about the incomplete composition of the teams, lack of professional ability and low financial resource were reported. This research gathers information for knowledge and analisys of the management actions of the nurses in the basic atention.

Key words: Nursing; Management; Basic health unit.

\section{RESUMEN}

Se objetivó analisar el trabajo del enfermero gerente, conocer sus acciones, comprobar la importancia asignada a la planificación y identificar factores que interferen en la dirección local. Pesquisa descriptiva, realizada em 10 unidades básicas de salud de Fortaleza, CE, Brasil, com 10 enfermeros, seleccionados Que tienen como oficio la gerencia. La recogida de datos ocurrió en enero de 2008 por intermedio de una entrevista estructurada. De esos, la mayoría era mujeres en la faja etaria de 37 a 57 años. Para los gerentes dirigir personas y la planificación es lo más importante en la dirección. Relataran dificuldades cuanto a la composición incompleta de los equipos, falta de capacitación profesional y la carencia de recursos financieros. Esta pesquisa reúne informaciones relevantes para conocimiento y análisis de las acciones de dirección del enfermero en la atención básica.

Descriptores: Enfermería; Gerencia; Unidad básica de salud. 


\section{INTRODUÇÃO}

A gerência constitui um importante instrumento para a efetivação das políticas de saúde, pois incorpora um caráter articulador e integrativo, em Que a ação gerencial é determinada e determinante do processo de organização dos serviços de saúde ${ }^{(1)}$.

Em cada organização é necessária a atuação de gerentes Que têm o papel de solucionar problemas, dimensionar recursos, planejar sua aplicação, desenvolver estratégias, efetuar diagnósticos de situações, garantir o desempenho de uma ou mais pessoas entre outras atividades Que são imprescindíveis para o desempenho da mesma $^{(2)}$.

Nesse contexto, a ação gerencial numa unidade básica de saúde caracteriza-se em grande parte pela análise do processo de trabalho, com identificação de problemas e busca de soluções para reorganização das práticas de saúde, na tentativa de alcançar as metas descritas no planejamento. Portanto, o gerente atua como interlocutor e mediador do processo de trabalho ${ }^{(3)}$.

É necessário a participação da academia e dos serviços, num esforço conjunto para rever as práticas e as intervenções necessárias, no âmbito gerencial, repensando as dicotomias existentes entre os propósitos e projetos de formação da força de trabalho em saúde e propósitos e projetos dos serviços, implicando em mudanças na visão de mundo, de idealista para realista ${ }^{(4)}$.

Com o advento do Programa de Saúde da Família como modelo de atenção básica e centro ordenador da rede de atenção à saúde do Sistema Único de Saúde (SUS) e sua grande expansão no país, é de suma importância Que se conheça e se defina um modelo de gerência para esta área. Este deve contar com planejamento adequado e um fluxo de atendimento sem grandes obstáculos para o usuário, sendo assim coerente com os princípios do programa ${ }^{(5)}$.

A enfermagem é uma das categorias da saúde mais mobilizadas para o gerenciamento das unidades básicas de saúde e cabe a essa o compromisso, junto aos demais profissionais, da viabilização do SUS, incentivando a participação da equipe na organização e produção de serviços de saúde para atender às reais necessidades dos usuários, trabalhadores e instituição. Para isso, deve utilizarse da descentralização administrativa, comunicação informal, flexibilidade na produção e estímulo à iniciativa e à criatividade de indivíduos e grupos ${ }^{(6)}$.

No espaço em Que ocorre a gerência exercida por enfermeiros, à relação desse profissional com o usuário e com outros trabalhadores existentes no cenário da saúde, são condições Que constituem e imprimem determinadas características no processo de trabalho desse profissional.

Dentre as competências gerenciais dos enfermeiros pode-se citar: análise crítica para tomada de decisão gerencial e o desenvolvimento do pensamento autônomo; organização de redes de serviços de saúde; desenvolvimento de instrumento para análise da situação de saúde e provisão de serviços e elaborar estratégias de intervenção; identificação de potencialidades e limitações institucionais Que diminuam ou impeçam a efetividade das ações de saúde; realização de planejamento e programação, fundamental à análise de situação e elaboração de propostas de intervenção. Utilização do sistema de informação, avaliando suas potencialidades e limitações; desenvolvimento dos conhecimentos gerenciais a partir de novos enfoques e modernas técnicas de gestão, entre outras ${ }^{(7,8)}$.
Sabe-se Que a maior parte da produção do gerenciamento em enfermagem está voltada ao gerenciamento de recursos humanos, entretanto, existem outras áreas Que não têm merecido a devida atenção desse profissional, como a área de custo, da regulação, da avaliação entre outras. Portanto, este estudo justifica-se pela carência e relevância da elaboração de peşuisas na área do gerenciamento da atenção básica e a participação do enfermeiro ${ }^{(9)}$.

No Brasil se gasta, anualmente, 80 dólares per capita com saúde, com significativo desperdício de recursos e a capacitação gerencial para definir e resolver problemas torna-se imprescindível na contenção e redirecionamento de gastos nos serviços de saúde ${ }^{(10)}$.

Assim, considerando Que a garantia da satisfação de trabalhadores e clientes, aliada à sobrevivência da organização é uma tarefa gerencial complexa, a análise do trabalho do enfermeiro gerente em organizações de atenção básica, é fundamental para o avanço das melhorias neste serviço. Esse estudo pode favorecer reflexões para futuras intervenções mais adęuadas voltadas à melhoria da gestão.

Objetivou-se analisar o trabalho do enfermeiro gerente de Unidade Básica de Saúde (UBS), no município de Fortaleza, CE e especificamente, descrever o perfil profissional dos enfermeiros gerentes, conhecer as ações gerenciais realizadas, verificar a importância atribuída pelos mesmos ao planejamento das atividades e identificar fatores Que facilitam e dificultam a realização das ações gerenciais do enfermeiro na Unidade Básica de Saúde.

\section{METODOLOGIA}

Pesquisa de abordagem Qualitativa desenvolvida em 10 UBS pertencentes a duas secretarias executivas regionais (SER) IV e V, em Fortaleza, CE.

Os sujeitos deste estudo foram enfermeiros, com vínculo funcional efetivo da Secretaria Municipal de Saúde de Fortaleza, exercendo a função de gerência/direção de Unidade Básica de Saúde, independente do tempo de exercício do cargo.

A amostra foi composta por 10 enfermeiros, de um total de 16 Que gerenciavam Unidades da área, sendo Quatro enfermeiros coordenadores de UBS da SER IV e seis que gerenciavam unidades da SER V.

O instrumento de coleta de dados foi à entrevista estruturada Que constava de perguntas abertas e fechadas, com informações acerca do perfil profissional, caracterização de atividades dos gerentes e Questões sobre o processo de trabalho dos mesmos. As entrevistas foram gravadas e transcritas conforme a permissão dos sujeitos.

A coleta de dados foi realizada em janeiro de 2008. Os enfermeiros foram abordados em seus respectivos locais de trabalho, em horário previamente acordado entre as partes. Os resultados foram apresentados por meio de tabelas e transcrições de falas dos entrevistados, com posterior análise.

O processo de coleta de dados iniciou-se após aprovação do Comitê de Ética da Universidade Estadual do Ceará e liberação dos campos para pesquisa, através do parecer $n^{\circ} .073942138$ FR CONEP 159829.

O presente estudo foi conduzido conforme a Resolução 196/ 96 da Comissão Nacional de Ética na Pesquisa, Que regulamenta as diretrizes e normas da pesQuisa envolvendo seres humanos. Dessa 
forma os sujeitos da pesquisa assinaram o termo de consentimento, após ter sido informado sobre objetivos, métodos, benefícios previstos ou potenciais riscos da peseuisa e garantido anonimato ${ }^{(1)}$.

\section{RESULTADOS E DISCUSSÃO}

Os dados são apresentados e analisados descritivamente. Optouse por organizá-los em duas partes. A primeira refere-se à apresentação dos enfermeiros, Que participaram do estudo, e a segunda aborda os processos de trabalho dos mesmos, no exercício do cargo de gerentes de unidades básicas de saúde.

\section{Perfil da população estudada}

Quanto ao perfil dos sujeitos, nove eram do sexo feminino. A maioria situava-se na faixa etária de 37 a 57 anos. Seis entrevistadas trabalhavam na instituição há até três anos e oito exerciam o cargo em média há três anos. Ao analisar-se o tempo de trabalho na Instituição e o tempo do exercício do cargo, verificou-se Que esse tempo é bem próximo na Quase totalidade dos gerentes, e seis entrevistados referiram, ainda, trabalho anterior na mesma função, o Que significa Que estes já possuem certa experiência no cargo Que ocupam.

Em relação à Qualificação dos gerentes, oito tinham realizado curso de especialização, sendo dois na área de educação e seis na área de gestão. A capacitação para o cargo, neste estudo, foi considerada Quando relacionada a cursos e/ou programas na área de gestão, administração e organização de serviço, especificamente, para a atenção básica. Com esta compreensão, seis afirmaram ter feito cursos específicos para o cargo.

Entretanto, para alguns autores a capacitação no âmbito da gestão e do gerenciamento em saúde e em enfermagem, ainda carece de fortalecimento das competências Que integram as dimensões éticas - políticas, Que compreendem a instância relacional e de responsabilidade social da profissão. Portanto, só a capacitação na área não é garantia de um bom trabalho de gerência ${ }^{(9)}$.

\section{Prática gerencial dos enfermeiros no cotidiano da Unidade}

Sobressai nessa seção a caracterização dos diferentes momentos do processo de trabalho dos gerentes, Que relacionam modos de pensar e agir. Enfoca, ainda, relações de poder e interpessoais, formais e informais, desses trabalhadores com as equipes e os usuários (Quadro I).

Dentre as atividades apontadas como importantes, a gestão de pessoas foi citada por sete como característica importante no gerenciamento das UBS, seguida pela gestão de processos e a gestão centrada no usuário.

No processo de trabalho da gestão, as habilidades sociais são hoje mais valorizadas Que as técnicas e o seu desenvolvimento fica mais presente Quanto mais alto na hierarQuia se encontra o gestor. A habilidade em gerenciar equipes é crucial para o gestor e, portanto, o investimento em desenvolvimento pessoal contínuo, provimento de senso de direção e estrutura, ajuda a equipe a gerenciar fronteiras, gerenciar o tipo e o tempo das intervenções ${ }^{(12)}$.

Para pensar e fazer o gerenciamento do trabalho em saúde, deve-se visualizar o ser humano, meio ambiente e a integração dos diversos saberes. A ordem deste tempo atual é de repensar novas alternativas, não radicais e sim integrativas, Que possam agregar

\begin{tabular}{|c|c|}
\hline $\begin{array}{c}\text { Atividades gerenciais de maior } \\
\text { importância }\end{array}$ & $\begin{array}{c}\text { Enfermeiros gerentes de } \\
\text { UBS }\end{array}$ \\
\hline Centradas na equipe & 7 \\
\hline Centradas nos processos & 6 \\
\hline Centradas nos usuários & 5 \\
\hline
\end{tabular}

Quadro 1. Visão dos gerentes Quanto à importância das atividades gerenciais executadas em Unidades Básicas de Saúde. Fortaleza, CE, 2008.

eficiência e, conseQüentemente, saúde aos seus profissionais e aos pacientes.

Conforme dados do Quadro 1, o trabalho gerencial centrado no usuário foi o menos citado, o Que demonstra ser necessário Que os gerentes compreendam Que a ação gerencial deve incluir o usuário, inclusive como ator e parceiro do processo gerencial, já Que a finalidade desta atividade é fornecer um cuidado qualificado a estes.

Em pesquisa realizada com enfermeiros gerentes da área básica, os enfermeiros consideram Que, dentro do processo de trabalho gerencial, manter o bom relacionamento com o usuário e com a comunidade faz parte da função gerencial como uma ação de respeito à cidadania e participação popular ${ }^{(13)}$.

Os gestores têm grande dificuldade para entender e para propor soluções viáveis para os problemas cotidianos do trabalho da gerência, o Que significa uma carência de compreensão mais profunda dos fundamentos dos sistemas de serviços de saúde e de seu papel ${ }^{(14)}$.

\section{Visão dos gerentes Quanto às dificuldades e facilidades no gerenciamento das atividades exercidas na UBS}

As principais dificuldades apontadas pelos entrevistados foram composição incompleta das equipes de PSF, falta de capacitação de alguns profissionais para o trabalho na unidade, falta de recursos financeiros, material e equipamentos para o exercício contínuo das atividades, além da dificuldade em referenciar usuários. Isso pode ser verificado nas seguintes falas:

\section{[...] Agora com o concurso vieram mais seis agentes comunitários de saúde, mas ainda falta... a gente tem bastante área descoberta... (G 4).}

[...] Falta de apoio financeiro às vezes... você faz um projeto pra desenvolver uma atividade grande né?, Que envolva a comunidade... você vai atrás de apoio a nível Central, a nível Regional e às vezes tem um bloqueio, ou por falta de verba ou não sei o quê que aí custa... (G 6).

[...] De dificuldade é a nossa demanda Que é grande e funcionário que é meio complicado... tem muita fofoquinha... muita besteirinha...entre funcionários, tanto nível médio, como nível superior (G7).

Pode-se destacar nas falas, Que as dificuldades citadas são as mesmas inerentes a todo o sistema de saúde, entretanto, é preciso repensar formas de superação dessas, para Que as mesmas não se tornem uma situação natural, previsível e inerente a área. 
Observando essas limitações, percebe-se a importância Que elas ocupam no trabalho cotidiano do gerente e o empenho desprendido por eles para a resolução dos problemas canalizando assim, a maior parte da sua atenção.

As situações Que favorecem o trabalho, conforme referiram os entrevistados foram: as relações interpessoais com a eQuipe, usuários e diversos setores da comunidade, além da identificação com o trabalho e o comprometimento da equipe.

Entretanto, essa integração da equipe é fundamental para a Estratégia Saúde da Família e, caso falhe, o modelo de atenção fragmenta, desumaniza, apresenta divisão rígida de trabalho e desigual valorização dos diferentes profissionais ${ }^{(15)}$.

De acordo com o Quadro 2, é referido pelos gerentes Que a importância do planejamento na ação gerencial relaciona-se, principalmente, a facilidade no alcance das metas e objetivos e ao controle e estímulo as atividades na UBS.

Planejar é o processo de analisar e entender um sistema, avaliar a sua capacidade, formular suas metas e objetivos, avaliar a efetividade dessas ações, ou planos, escolher as prioridades, iniciar as ações necessárias para sua implantação e estabelecer um monitoramento contínuo do sistema, a fim de atingir um nível ótimo de relacionamento entre o plano e o sistema ${ }^{(16)}$.

No depoimento dos entrevistados também foram detectadas etapas para a formulação do planejamento, como diagnóstico, prioridades, metas, recursos, discussão entre funcionários, execução, resultados, avaliação das ações e reformulação de ações, como podemos observar a seguir:

[...] A gente divide de acordo com as épocas. Estamos numa Quadra invernosa agora, então a gente programa para os tipos de doenças oportunistas nesse período, né?..., Que são as viroses, doenças respiratórias e tudo (G8).

[...] Fizemos o mutirão da dengue, em Que a população veio doar lixos recicláveis na unidade... vendemos esse lixo coletado pras indústrias de reciclagem de lixo, e, com o dinheiro, montamos uma biblioteca para a comunidade, e muita gente vem fazer empréstimo de livro com a gente, alunos vêm fazer pesquisas... Isso serviu pra combate à doença, como também pra enriQuecimento cultural à população...(G5).

[...] Você vai aprendendo, esse planejamento a cada ano vai melhorando... tá tudo muito avançado...(G3).

A função gerencial tem como propósito o desenvolvimento e a eficiência organizacional. No caso da saúde, o gerente deve utilizar o conhecimento para planejar, programar, desenvolver, controlar as atividades realizadas nas unidades básicas de saúde, cumprindo com a missão social e humana, promovendo e protegendo a saúde da população ${ }^{(17)}$. Portanto os enfermeiros do estudo, em suas falas, referem valorizar o planejamento como um instrumento importante da prática da gestão, bem como adequá-lo as especificidades de cada situação.

Quanto à solicitação de ajuda para a elaboração do planejamento, as falas reportadas abaixo confirmam a presença de coordenação de programas, chefias e profissionais envolvidos para resolução de problemas.

\begin{tabular}{|c|c|}
\hline $\begin{array}{l}\text { Unidades Básicas de } \\
\text { Saúde }\end{array}$ & $\begin{array}{l}\text { Justificativa da importância do } \\
\text { planejamento }\end{array}$ \\
\hline l & Nortear ações. \\
\hline 3 & Alcançar metas e objetivos. \\
\hline 3 & Controlar e estimular atividades. \\
\hline l & Facilitar a execução das atividades. \\
\hline 1 & Atendimento da demanda. \\
\hline 1 & $\begin{array}{l}\text { Reuniões com as equipes de PSF para } \\
\text { percepcão de necessidades }\end{array}$ \\
\hline
\end{tabular}

Quadro 2. Importância atribuída pelos enfermeiros ao planejamento na prática da gerência. Fortaleza, 2008.

[... Foram realizadas várias ajudas, inclusive a Regional..., apesar dos funcionários também terem contribuído muito (G2).

[...] A ajuda vem dos cursos de capacitação profissional Que a gente faz, né?... Temos também um "tutor", ligado ao Ministério da Saúde, e a gente vai aprendendo... Pedimos ajuda às enfermeiras também, né?... (G3).

A utilização de recursos de forma sistematizada, aliada à participação comunitária inexiste na elaboração do planejamento e é contrária à inovação, já Que constitui uma opção de planejamento normativo. No entanto, há um caráter dinâmico, flexível, atrelado à conjuntura do planejamento nos depoimentos dos gerentes. Eles reconhecem Que o planejamento envolve o enfrentamento de forças sociais, Que seria o enfoQue estratégico da programação em saúde. A ação dos grupos sociais e o significado Que adQuire a interação entre eles através dos aspectos formais, materiais ou organizativos podem, pelo seu caráter "objetivo", ser tratados na ação do planejamento $^{(18)}$.

No entanto, para Que o gerente comande com eficiência é necessário planejar, isto é, determinar aonde se Quer chegar, sendo importante a clareza da missão da unidade, objeto que deve ser construído com toda a equipe. É o planejamento Que vai nortear o processo de tomada de decisões Que levará a meta desejada ${ }^{(8)}$.

\section{CONSIDERAÇÕES FINAIS}

Os dados relativos ao perfil e Qualificação, dos sujeitos do estudo, indicaram Que os gerentes de unidades possuem certa experiência no cargo Que ocupam.

Em relação às atividades gerenciais referidas como importantes, destacou-se a gestão de pessoas, seguida pela gestão de processos e posteriormente, a centrada no usuário, demonstrando ser necessária a compreensão, pelos gerentes, da importância da participação do usuário como ator social e parceiro no processo gerencial.

Dentre as dificuldades apontadas pelos entrevistados, na execução do trabalho gerencial, foram citadas composição incompleta das equipes de PSF, falta de capacitação de alguns profissionais para o trabalho na unidade, falta de recursos financeiros, material e equipamentos, entretanto, é preciso repensar formas de superação dessas. As situações Que favorecem o trabalho da gerência foram às relações interpessoais com a equipe, usuários e diversos setores da comunidade, além da identificação com o 
trabalho e o comprometimento da equipe.

O planejamento das ações é realizado pelos gerentes Que atribuem a este importância relacionada, principalmente, a facilidade no alcance das metas e objetivos e ao controle e estímulo as atividades na UBS.

Conclui-se Que, a pesquisa apresentada reúne informações relevantes para o conhecimento e análise das ações do processo de trabalho gerencial do enfermeiro na Unidade Básica de Saúde. Embora considerando que o presente estudo contribui para o conhecimento do perfil, considera-se a necessidade de estudos Que ampliem as fontes de coleta de dados e permitam trabalhar outros elementos de análise da gerência de enfermagem nessa área.

\section{REFERÊNCIAS}

I. Ciampone MHT, Kurcgant P. O ensino da administração em enfermagem no Brasil: o processo de construção de competências gerenciais. Rev Bras Enferm 2004; 57(4): 40 I-7.

2. Chiavenato I. Introdução à teoria geral de administração. São Paulo: McGaw-Hill; 2000.

3. Santos ASS, Miranda SMRC, organizadores. A enfermagem na gestão em atenção primária à saúde. Barueri: Manole; 2007.

4. Lunardi FDW, Lunardi VL. Uma nova abordagem no ensino da enfermagem e de administração em enfermagem como estratégia de (re)orientação prática profissional do enfermeiro. Texto Contexto Enferm 1996; 5(2): 20-34.

5. Santos RC. Saúde todo dia: uma construção coletiva. São Paulo: Hucitec; 2006.

6. Guimarães EMP, Évora YDM. Sistema de informação: instrumento para tomada de decisão no exercício da gerência. Cien Inf 2004; 33(1): 72-80.

7. Fracolli LA, Egry EY. Processo de trabalho de gerência: instrumento potente para operar mudanças nas práticas de saúde? Rev Latino-am Enfermagem 2001; 9(5): 13-8.

8. Miranda SMRC. Gerenciamento da unidade básica de saúde: a experiência do enfermeiro. In: Santos AS, Miranda SMRC, organizadores. A enfermagem na gestão em atenção primária à saúde. Barueri: Manole; 2006. p. 81-122.

9. Kurcgant P, Ciampone MT. A pesquisa na área de gerenciamento em enfermagem no Brasil. Rev Bras Enferm 2005; 58(2): 16 I-4.

10. Júnior GDG, Vieira MMF. Qualidade total e administração hospitalar: explorando disjunções conceituais. Ciênc Saúde
Coletiva 2002; 7(2): 325-34

1 I. Ministério da Saúde (BR). Conselho Nacional de Saúde. Comitê Nacional de Ética em Pesquisa em Seres Humanos. Resolução no 196 de 10 de outubro de 1996: diretrizes e normas regulamentadoras de pesQuisa envolvendo seres humanos. Brasília: Ministério da Saúde; 1996.

12. André AM. Competências para a gestão de unidades básicas de saúde: percepção do gestor [dissertação]. São Paulo: Escola de Enfermagem, Universidade de São Paulo; 2006.

13. Weirich CF, Munari DB, Mishima SM, Bezerra ALQ. O trabalho gerencial do enfermeiro na Rede Básica de Saúde. Texto Contexto Enferm 2009; 18(2): 249-57.

14. Mendes EV. Os sistemas de serviços de saúde: o que os gestores deveriam saber sobre essas organizações complexas. Fortaleza: Escola de Saúde Pública do Ceará; 2002.

15. Vieira C, Cordeiro M. Trabalho e subjetividade: intervenção psicossocial com uma equipe de profissionais do Programa Saúde da Família. Are Bras Psicol 2006; 57(I): 58-74.

16. Passos IP. A utilização de indicadores na prática gerencial do enfermeiro em Unidades Básicas de Saúde da cidade do Rio de Janeiro [tese]. São Paulo: Escola de Enfermagem, Universidade de São Paulo; 2004

17. Lazzarotto EM. Competências essenciais requeridas para o Gerenciamento de unidades básicas de saúde [dissertação]. Florianópolis: Pós-Graduação em Engenharia de Produção, Universidade Federal de Santa Catarina; 2001.

18. Rocha JSY. Análise crítica dos modelos de planejamento em saúde na América Latina. Educ Med Salud 1992; 26(2). 